\title{
Transoral approach to the cervical spine: report of four cases
}

\author{
SA O'LAOIRE,* DGT THOMAS $\dagger$ \\ From the Atkinson Morley's Hospital* and the National Hospital, Queen Square, † London
}

SUMMARY The transoral approach to the upper cervical spine is an established but little used route, offering excellent access with good wound healing, to lesions of the bodies of the atlas, axis and upper part of the third cervical vertebra. The authors report four cases which demonstrate the value of the procedure.

Surgery for disorders of the cervical spine anterior to the cord is commonly performed through the anterior route, between the sternomastoid muscle and carotid sheath on the one hand, and the trachea and oesophagus on the other. However, the inaccessibility of the spine through such a route in the upper levels has generally resulted in the less satisfactory posterior approach, with attendant dangers to the spinal cord and medulla. An alternative anterior approach through the thin, relatively avascular midline raphe of the pharynx was proposed nearly twenty years ago, ${ }^{1}$ but there have been relatively few reports since then, ${ }^{2-9}$ and there are no more than thirty cases reported in the literature. The authors report four transoral operations on the cervical spine, including three cases of atlantoaxial dislocation and one of cord compression by tumour.

The operative technique was based on that of Fang and Ongl with use of the operating microscope. The patients were anaesthetised and intubated, skull traction was applied and a tracheostomy performed. The tongue was retracted with a BoyleDavies gag, and the uvula with a suture to the hard palate.

A four centimetre midline incision was made in the posterior pharyngeal wall with its upper third at the anterior tubercle of the atlas (which is readily palpable). The soft tissues were stripped laterally off the anterior aspects of the vertebral bodies and intervertebral discs, and the muco-muscular flaps

Address for reprint requests: SA O'Laoire, Atkinson Morley's Hospital, 31 Copse Hill, Wimbledon SW20 ONE, UK.

Received 27 March 1981 and in revised form 6 September 1981

Accepted 26 September 1981 so raised were then held apart with stay sutures. Bone removal was performed with curettes and air drill in patients with hard bone consistency; pituitary rongeurs were often sufficient when bone was softened by tumour or rheumatoid disease. Peroperative plain radiographs or screening was used to guide the surgeon in the extent of bone removal. Fusion was effected between the lateral masses (when necessary) using chips of the removed bone placed between the articular facets of the lateral intervertebral joints, after these had been denuded of cartilage. The pharynx was repaired with a two layer closure using absorbable suture material. Oral nourishment was withheld for five days. A nasogastric tube was not used. A throat swab was taken before operation, and prophylactic antibiotic cover with flucloxacillin and sulphadimidine was administered for ten days after operation.

\section{Case reports}

Case 1 Mrs SB was a 64-year-old woman who had suffered severe head, chest and abdominal injuries in a road traffic accident 4 months previously. On regaining consciousness 4 weeks after injury she complained of severe pain in her neck, which persisted and which was associated during rehabilitation with sudden transient episodes of weakness of her arms and legs. Plain radiographs of the spine showed a fracture dislocation of the first and second (C1 and $\mathrm{C} 2$ ) cervical vertebrae. Myelography showed anterior compression of the cord by displacement of part of $\mathrm{C} 2$ body (fig 1). At operation the anterior arch of $\mathrm{Cl}$, the dens and upper two-thirds of $\mathrm{C} 2$ body were removed and a lateral fusion was performed. The patient spent three weeks on traction and three months in a cervical collar. She made an excellent recovery, losing her neck pain and having no further drop attacks. Sound bony fusion was evident on radiographs obtained 3 months after operation (fig 2 ). 


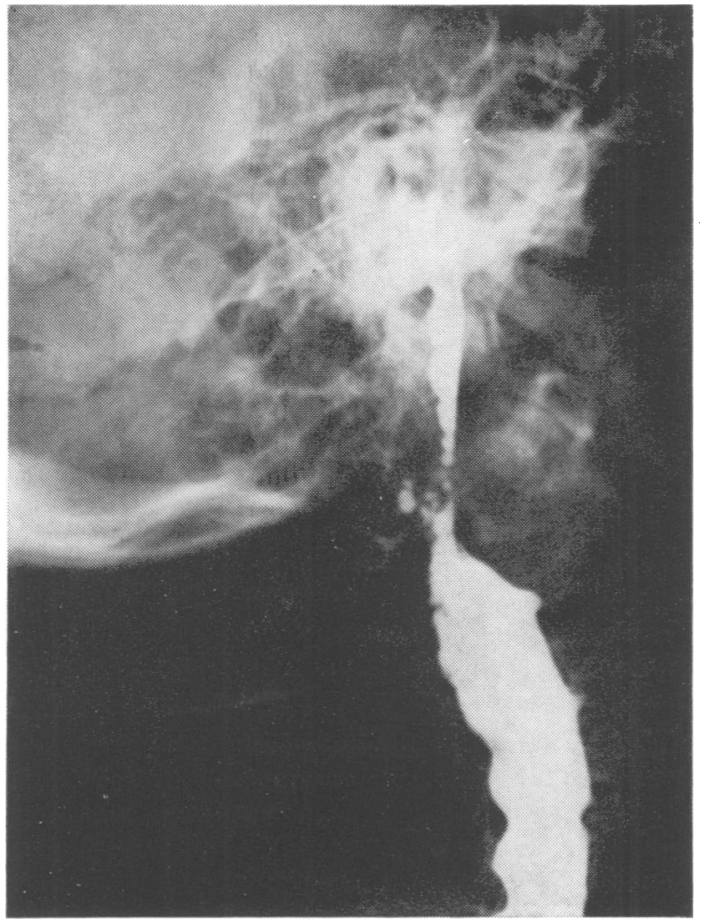

Fig 1 Myelogram showing posterior displacement of the body of C2 (Case 1).

Case 2 Mr RC aged 51 years had a 30 year history of rheumatoid arthritis. He developed a spastic tetraparesis and bulbar palsy causing recurrent aspiration pneumonitis, owing to atlanto-axial dislocation. Following a posterior fusion (occipito-cervical) without decompression 3 years previously he had improved, but subsequently presented with an 18 month history of recurrent symptoms. Examination revealed a spastic bulbar palsy causing constant inhalation of fluids and food, and a symmetrical tetraparesis of MRC grade 4/4+, with spasticity so severe that he required the use of two canes for walking and required help for feeding and dressing. Radiographs showed atlanto-axial dislocation, and myelography showed severe anterior compression by posterior tilt of the odontoid process. At operation the odontoid process was exposed by removing the anterior arch of the atlas and was removed. Fusion was then performed between the lateral masses. He regained virtually normal swallowing (no inhalation of food, but occasionally food "sticks"), is walking without help, is independent in feeding and dressing, and has returned to work.

Case 3 Mrs EM was a 71-year-old woman who had rheumatoid disease for 25 years. She presented with a 3 year history of progressive weakness of the arms and legs, which had not been influenced by an occipitocervical fusion (without decompression) 2 years

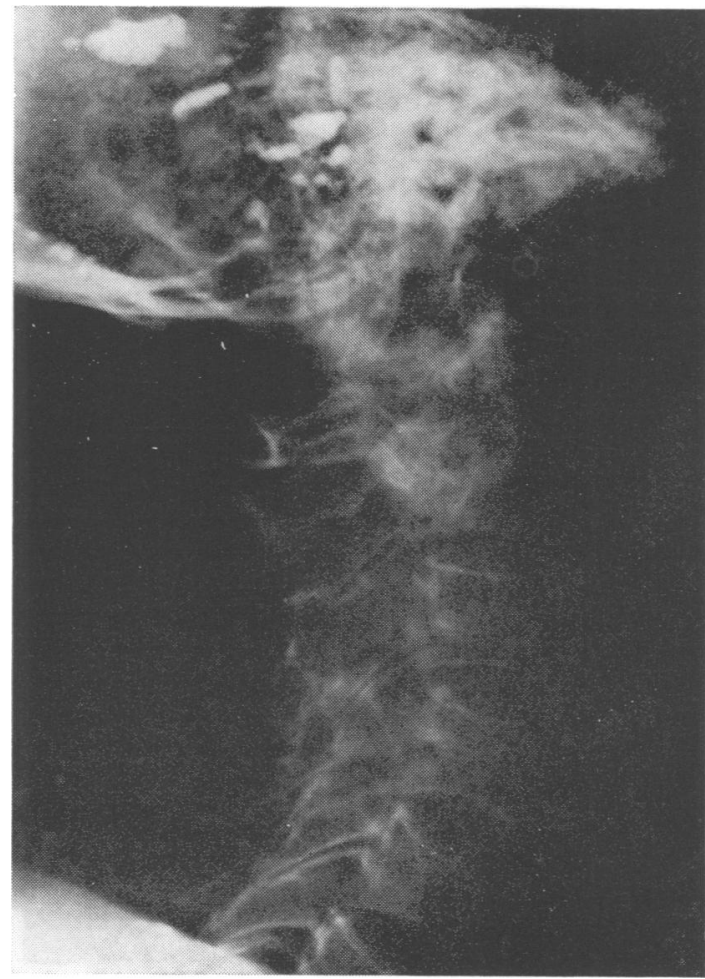

Fig 2 Plain radiograph three months after operation showing bony fusion of C1 and C2 (Case 1).

earlier. She was severely disabled by a tetraparesis so advanced that voluntary movement was present only in the left upper limb (MRC 3), with which she could use a spoon for feeding. Plain radiographic and myelographic findings (fig 3) were identical to those of Case 2, as was the operative procedure. She made a rapid neurological improvement, regaining full strength in the left upper limb, and recovering movement in her legs (MRC 3). Unfortunately she developed pneumonia and died three weeks after operation.

Case 4 CM was an 8-year-old boy who 2 years previously complained of neck pain. Radiographs at that time showed abnormality of the second and third cervical vertebrae. Biopsy via the posterior route revealed eosinophilic granuloma. He was treated with local radiotherapy and the pain resolved. He subsequently represented with a 2 month history of progressive weakness of the left limbs and return of neck pain. Examination showed a moderately severe (MRC grade III) left limb weakness and bilateral extensor plantar responses. Radiographs showed destructive change in the second and third cervical vertebrae; a myelogram (fig 4) showed an extradural mass, which was shown to be relatively avascular by spinal angiography. At operation the body of $\mathrm{C} 2$ and most of $\mathrm{C} 3$ was excised together with 


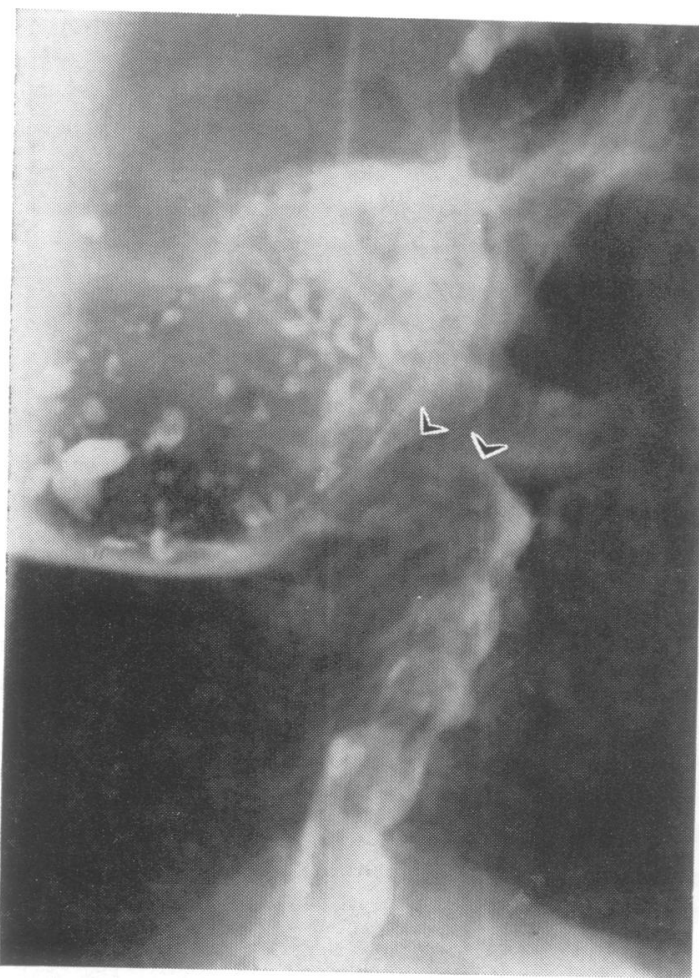

Fig 3 Myelogram showing anterior compression by atlanto-axial dislocation (Case 3). (The head is in a position of fixed extension following previous posterior fusion.)

extradural tumour. The lateral vertebral elements were stable and a graft was therefore not performed. Power improved greatly after operation (MRC IV + ) and further radiotherapy and chemotherapy was given. He remained well until he died about 18 months later from pulmonary embolus.

\section{Discussion}

Transoral operation on the cervical spine is not often indicated; anteriorly placed compression of the upper spinal cord and lower medulla by tumour is not common (there are four reported cases $^{2-6}$ ) and the posterior approach for the relatively common atlanto-axial dislocation of rheumatoid origin usually works well, although the extensive occipito-cervical fusion required abolishes all movement of the head upon the neck. ${ }^{5}$ Rotation of the head is preserved following atlanto-axial fusion by whatever route, but the success rate of interlaminar and interspinous fusion is poor $^{5}$ and the transoral operation would appear to be the procedure of choice when preservation of head

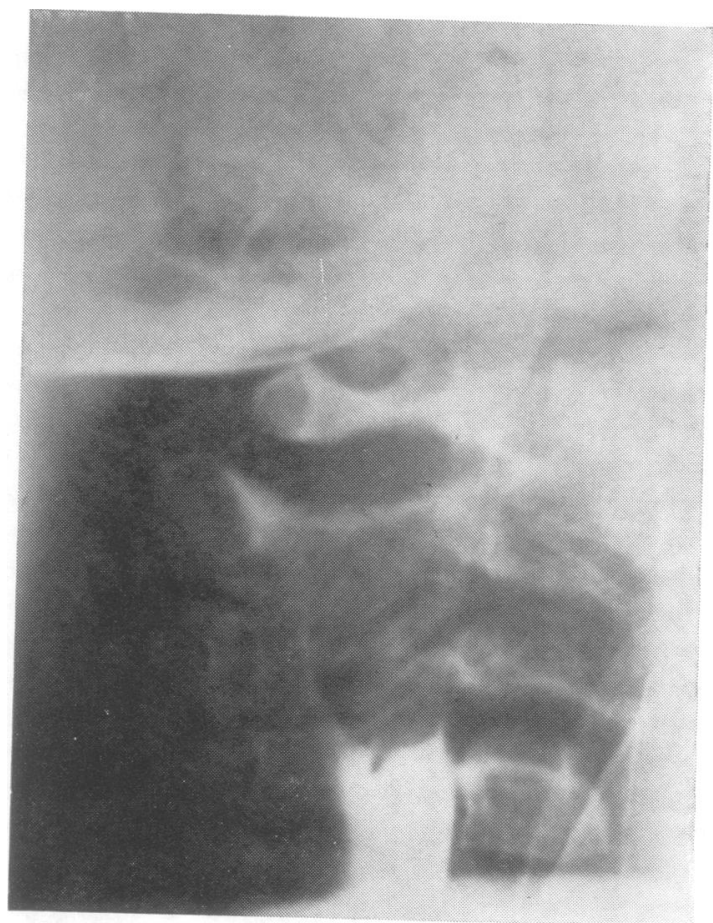

Fig 4 Myelogram showing destructive change in $C 2$ and $C 3$ bodies; anterior intraspinal mass (Case 4).

rotation is desired. Atlanto-axial dislocation due to trauma has been treated by the transoral route in 10 reported cases 135 with successful outcome, as in our case. Recurrent spontaneous dislocation due to hypoplasia of the os odontoideum has also been treated successfully, ${ }^{14}$ and there have been individual reports of transoral operation for spinal cord compression due to tuberculous osteomyelitis ${ }^{1}$ and Paget's disease. ${ }^{6}$

The transoral approach to the cervical spine appears to be safe and effective. The pharyngeal wound heals well. Cases of infection in early reports were associated with the presence of a nasogastric tube overlying the wound, ${ }^{1}$ with inadvertent opening of the spinal theca, ${ }^{1}$ or with insertion of large onlay bone grafts under the pharyngeal incision. ${ }^{2} 35$ Our experience suggests that a sound fusion can be obtained by inserting bone chips from the operative site between the articular facets; a second incision is avoided, and closure of the pharyngeal wound without tension is more easily achieved without a large midline onlay graft. Damage has occurred to the vertebral artery ${ }^{1}$ and that risk should be avoided by the exercise of great care during preparation of the articular facets for fusion.

Temporary tracheostomy has been part of the 
standard management, ${ }^{249}$ but the transoral operation has been performed without per-operative or post operative difficulty using a standard oraltracheal tube. ${ }^{3}$ Late (two months after operation) respiratory obstruction has occurred in children from polyp formation at the tracheostomy site, ${ }^{1}$ and it would seem advisable to avoid tracheostomy in childhood.

The authors consider that the transoral approach should be considered when appropriate; in cases of anteriorly placed masses in the atlas, axis and upper part of the third cervical vertebral body; following traumatic atlanto-axial dislocation; and in rheumatoid disease when compression of the cord and medulla is anteriorly placed (including recurrent compression following posterior fusion). The approach gives excellent access, and with the illumination and magnification of the operating microscope is not technically difficult.

\section{References}

${ }^{1}$ Fang HSY, Ong GB. Direct approach to the upper cervical spine. J Bone Joint Surg (Am) 1962;44:1 588-604.
${ }^{2}$ Mullan S, Naunton R, Hekmat-Panah J, Vailati G. The use of an anterior approach to ventrally placed tumors in the foramen magnum and vertebral column. $J$ Neurosurg 1966;24:536-43.

${ }^{3}$ Estridge MN, Smith RA. Transoral fusion of odontoid fracture. J Neurosurg 1967;27:462-5.

${ }^{4}$ Greenberg AD, Scoville WB, Davey LM. Transoral decompression of atlanto-axial dislocation due to odontoid hypoplasia. J Neurosurg 1968;28:266-9.

5 Thompson H. Transpharyngeal fusion of the upper cervical spine. Proc $R$ Soc Med 1970;63:893-6.

${ }^{6}$ Bonney G. Stabilization of the upper cervical spine by the transpharyngeal route. Proc $R$ Soc Med 1970; 63:896-7.

' Harris P. Atlanto-axial fracture dislocation treated by reduction and transoral bony fusion. Procs 17th Spinal Cord Injury Conference of the Veterans Administration, VA Hospital, Bronx, New York, 1969;17:40-5.

${ }^{8}$ Sukoff MH, Kadin MM, Moran T. Transoral decompression for myelopathy caused by rheumatoid arthritis of the cervical spine. $J$ Neurosurg 1972;37: 493-7.

${ }^{9}$ Apuzzo MJL, Weiss MH, Heiden JS. Transoral exposure of the atlanto-axial region. Neurosurgery $1978 ; 3: 201-6$. 


\section{Notices}

\section{The Volvo awards for low-back pain research}

In order to encourage research in low-back pain, the Volvo Company of Göteborg, Sweden, for 1983 has sponsored three prizes of $\$ 5,000$ each. Awards will be made competitively on the basis of scientific merit in the area of clinical studies, basic science and engineering.

Papers entering the contest must contain original material not previously submitted for publication. A multiple authorship is acceptable. The manuscripts, in the English language, should be full-length, in a form suitable for submission as an original paper (not a thesis) to a scientific journal. Five copies of each paper in full must reach the address given below, not later than December 10, 1982.

One of the authors should be prepared to come to Cambridge, England at the time of the meeting of the International Society for the Study of the Lumbar Spine, April
$7-10,1983$, to present the paper and to receive the prize.

The board of referees will be chaired by Professor Alf Nachemson, to whom all correspondence should be addressed, and will contain members from the fields of clinical medicine, bioengineering and biochemistry.

$$
\begin{array}{r}
\text { PROFESSOR ALF NACHEMSON } \\
\text { Department of Orthopaedic Surgery I, } \\
\text { Sahlgren Hospital, } \\
S-41345 \text { GÖTEBORG, Sweden }
\end{array}
$$

An International Conference on Recent Advances in Neurotraumatology will be held in Edinburgh under the auspices of the Neurotraumatology Committee of the World Federation of Neurosurgical Societies, 20-22 September, 1982. Further information may be obtained from Douglas Crawford, The Polecon Company Ltd, 22A Rutland Square, Edinburgh EH1 2BB, Scotland.
A Consensus Statement on Computed Tomographic Scanning of the Brain is now available from the Office for Medical Applications of Research, National Institutes of Health. Free, single copies may be obtained from Michael Bernstein, Office for Medical Applications of Research, Building 1, Room 216, National Institutes of Health, Bethesda, Maryland 20205, USA.

\section{Correction}

Transoral approach to the cervical spinereport of four cases by SA O'Laoire, DGT Thomas (J Neurol Neurosurg Psychiatry 1982;45:60-63). In the second sentence under Discussion, the authors intended to state that "Flexion of the head is preserved following atlanto-axial fusion by whatever route" (not "rotation of the head"). 